\title{
Strategische Aspekte der katholischen Medienarbeit ${ }^{*}$
}

\section{Vorbemerkung}

Die modernen Medien in ihrer gewachsenen und noch weiter wachsenden Vielfalt, Unterschiedlichkeit und Reichweite durchdringen alle Lebensbereiche der Menschen in allen gesellschaftlichen Gruppierungen und in jedem Alter. Wir haben es mit einer raschen Entwicklung zu tun. Längst sind die Instrumente der sozialen Kommunikation ein Konstitutivum der modernen Gesellschaft. Medien und Kommunikationsphänomene sind darin hochrelevante Größen.

Kirchliche Medienarbeit bekommt vor diesem Hintergrund eine sehr hoch einzuschätzende Bedeutung. Kirche ist integraler Teil der Gesellschaft und vielfältig in deren Kommunikationsprozesse hineinverwoben. Immer schon kommuniziert sie auch über Medien mit den Menschen. Es ist ihr Auftrag, zu den Menschen zu gehen. Medienarbeit erfüllt daher auch einen Verkündigungsauftrag. Dabei darf das Verständnis von „Verkündigung" nicht zu eng gefasst werden. Die mediale Kommunikation der Kirche weist ein weites Spektrum auf, zu dem Glaubensverkündigung und geistliche Angebote ebenso gehören wie die Information über kirchliche Themen, die Transparenz in finanziellen Fragen, die aktuelle politische Auseinandersetzung etwa bei ethischen oder sozialen Problemen oder das sozialanwaltschaftliche Eintreten für benachteiligte Personengruppen.

Wie weit dies in einem Zusammenhang mit dem „Verkündigungs"Auftrag der Kirche steht, bedürfte einer eigenen theologischen Reflexion. Grundsätzlich gilt das Ziel, eine möglichst große Zahl von Menschen anzusprechen und sich ihnen als einladende, glaubwürdige, „interessante" Kirche zu erweisen. Und dies mit den Mitteln moderner Kommunikationstechnologie und auf hohem qualitativem Niveau.

Aber wen erreicht die Kirche über die medialen Offerten? Aufschluss darïber gibt die Grundlagenforschung der Medien-Dienstleistung GmbH (MDG). Aus der "Milieustudie" von 2006 geht hervor, dass die Kirche über die "personale" Kommunikation - also durch

* Vortrag anlässlich des 40-jährigen Bestehens von Communicatio Socialis am 31. Januar 2008 in Eichstätt. 
Predigt, Glaubensgespräche, kirchliche Erwachsenenbildung sowie durch persönliche Begegnungen - nur eine begrenzte $\mathrm{Zahl}$ von $\mathrm{Ka}$ tholiken erreicht. Von den Befragten gaben 12 Prozent an, "häufig" Kontakt mit der Kirche zu haben, für 28 Prozent gilt dies nur „ab und zu“. Was die mediale Kommunikation anbelangt, so haben wir mit unseren kirchlichen Medien vor allem Zugang zu eher traditionell geprägten Personenkreisen, die in den letzten Jahrzehnten stark abgenommen haben und weiter abnehmen werden. Für den Zugang zu den sogenannten gesellschaftlichen "Leitmilieus" sind die Hürden hoch.

Katholische Medienkommunikation steht heute mehr denn je in einem harten Wettbewerb mit anderen Anbietern. Und in diesem Wettbewerb ist derjenige erfolgreicher, dem es gelingt, die Köpfe und Herzen, die Interessen und die emotionalen Bedürfnisse der Menschen besser anzusprechen. Dass dies oft sehr vordergründig geschieht, ändert nichts daran, sondern ist allenfalls eine Herausforderung an die kirchliche Medienkommunikation, auf die inhaltliche Konsistenz ihrer Angebote zu achten und zugleich darauf, dass diese dem Bedarf der gewünschten Rezipienten auch wirklich entgegenkommen. Die mangelnde Akzeptanz katholischer Medienkommunikation hat wohl auch damit zu tun, dass die darin praktizierte Art, "Sinnangebote" zu machen, nicht immer mit ausreichender Rückbindung an die Vorstellungen der Menschen entwickelt wurde, die sie erreichen will.

Anders gesagt: Es geht um die mediale und kommunikative „Anschlussfähigkeit" der Kirche an die unterschiedlichen Vorstellungen verschiedener Gesellschaftsgruppen im Hinblick auf Lebenssinn, Weltanschauung, Sprache und Ästhetik oder Stilistik. „Rezipienten" treffen eine aktive und völlig freie Entscheidung darüber, was sie aufnehmen und annehmen, wofür sie sich interessieren und wofür nicht. Sie sind eben nicht „Zielgruppen“, d.h. passive Empfänger unserer „Botschaften“. Wie können Menschen motiviert werden, unsere Angebote anzunehmen? Dies ist die Grundanfrage an jede Strategie kirchlicher Medienkommunikation.

Dabei besteht die enorme Aufgabe und Schwierigkeit zugleich darin, dass die Kirche von ihrem innersten Auftrag her eine Kirche für alle Menschen ist, weil das durch sie zu verkündende Heil universal und nicht exklusiv ist. Jede Bevorzugung bestimmter "Milieus", seien sie konservativ, aufgeklärt-progressiv, hedonistisch oder wie auch immer, widerspricht diesem Selbstverständnis der Kirche. Und zugleich muss es ihr gelingen, in einer modernen Gesellschaft Gehör zu finden, deren Fragen, Hoffnungen, Sehnsucht in ihrer ganzen pluralen Differenziertheit und Unterschiedlichkeit, ja Widersprüchlich- 
keit, anzunehmen und ernst zu nehmen. Es muss ihr auch gelingen, den Menschen in den sogenannten "Leitmilieus" dieser Gesellschaft eine einladende und glaubwürdige Gesprächspartnerin zu sein, damit sich ihr die Personengruppen öffnen, die die Zukunft der Gesellschaft maßgeblich mitgestalten.

Letzteres scheint heute wieder aussichtsreicher zu sein als noch vor wenigen Jahren. Der jüngst veröffentlichte „Religionsmonitor" der Bertelsmann-Stiftung erteilt der These eine Absage, dass es mit dem Religiösen bergab gehe. Im Gegenteil: Es ist wieder eine verstärkte Aufmerksamkeit für Religion zu beobachten. Auch für junge Menschen gilt dies. Von den 18- bis 29.Jährigen in Deutschland bezeichnen sich nach Auskunft der Bertelsmann-Studie 52 Prozent als "religiös" und 14 Prozent als „hochreligiös". Allerdings besagt dies keinesweg schon eine Identifizierung mit kirchlich geprägtem Christentum. Was unter "Religion" jeweils verstanden wird, weist ein mehr als buntes Bild auf. Und doch: Die Verehrung des „unbekannten Gottes" als Ort von Offenbarung - ist nicht die Areopag-Rede des Apostels Paulus in der Apostelgeschichte von jeher ein Vorbild dafür?

Ein Spagat also nach vielen Richtungen! Und indem ich die Fragestellungen benenne, verfüge ich noch keinesfalls über vollständig befriedigende Antworten. Auch die selbstkritische Reflexion gehört zu den Grundlagen einer Medienstrategie der katholischen Kirche.

\section{Ausgangslage}

In Zeiten knapper werdender Ressourcen ist eine stärkere strategiegeleitete Strukturierung kirchlichen Medienengagements erforderlich. Die katholische Kirche ist in verschiedenen Unternehmungen und Institutionen vertreten bzw. unterstützt diese. Systematisiert man die Funktionen der einzelnen Medieneinrichtungen, kommt man zu einem Drei-Säulen-Modell kirchlicher Medieninstitutionen:

- Katholisches Radio-, Fernseh- und Internet-Engagement

- Katholische Multiplikatorendienste

- Katholisches Print-Engagement.

Die Aufgabe der Kirche ist es, innerhalb dieser drei Säulen die Medienarbeit effektiv zu gestalten. Dabei sind Veränderungen zu benicksichtigen, die sich aus der sogenannten Sinus-Milieu-Studie ergeben.

In allen drei Bereichen gibt es derzeit Handlungsbedarf. Die zentrale Herausfordenung besteht darin, dass die Medienkommunikation in kirchlicher (Mit-) Verantwortung gesellschaftlich ,anschlussfähig“ und menschendienlich wird. Dies schließt im besonderen mit ein, dass die religiös relevanten Themen, die dem menschlichen Bedürfnis 
nach Sinnorientierung dienen, in der Medienwelt ihre angemessene Berïcksichtigung finden, sei es durch kircheneigene Angebote oder durch Offerten, die von einem religiös-spirituellen Engagement geprägt sind und aus Sicht der Kirchen Unterstiutzung verdienen.

Kirchliches Engagement muss dabei nicht nur den Wandel im Medienbereich begleiten, um zu entscheiden, wie ein kirchlicher Beitrag aussehen muss, der die Ziele einer menschendienlichen Kommunikation verwirklicht. Ein solches Engagement muss zwangsläufig auch mit Blick auf die Frage nach der finanziellen Realisierbarkeit erörtert werden.

In der derzeitigen Situation steht die Kirche vor großen Herausforderungen. In der Medienlandschaft zeichnet sich zurzeit ein Paradigmenwechsel $a b$, der durch den Umstieg von analoger zu digitaler Technik bestimmt ist. Dies fordert die Kirche heraus, sich mit den Auswirkungen der Digitalisienung zu befassen und neue Optionen eines kirchlichen Engagements in der digitalen Medienwelt zu prüfen.

Gleichzeitig dürfen traditionelle Engagements etwa im Printbereich nicht vernachlässigt werden, denn die Erfahnungen mit Innovationen im Medienbereich zeigen, dass neue Medien die alten nie vollständig ersetzen. Allerdings ist auch hier kritische Überprïfung angezeigt. Insgesamt muss die Kirche in dieser komplizierten Situation eine diversifizierte Strategie verfolgen.

Die Kirche kann sich aber nicht nur auf das eigene Medienangebot beschränken. Sie muss an der Lösung von Problemen im gesamten Medienbereich aktiv mitwirken. Im vergangenen Jahr ist etwa die öffentliche Debatte über die schädlichen Wirkungen von Medien voll entbrannt. Im Zentrum dieser Diskussion standen vor allem gewaltbesetzte Computerspiele, so genannte "Killerspiele“. Die Kirche muss ihre Grundorientierungen, ihren Wertekanon und ihre Kompetenz in diese Auseinandersetzungen einbringen. Die Probleme, um die es geht, kann ich hier nur andeuten: Ich denke zum Beispiel an die Globalisierung auch der medialen Märkte, an unterschiedliche und zum Teil einander widersprechende ethische Konzepte der Medienarbeit, an unterschiedliche Gesetzeslagen in den verschiedenen Ländern. Es geht aber auch hierzulande um medienpolitische Grundoptionen mit ethischen Konsequenzen. So etwa um die Frage: Ist Fernsehen ein Kulturgut oder ein reines Wirtschaftsunternehmen? Auf diese Frage müssen sowohl öffentlich-rechtliche als auch privat-kommerzielle Sendeanstalten eine Antwort finden. Die Antwort wird unterschiedlich ausfallen, aber sie hat in jedem Fall weitreichende Folgen.

Im Folgenden werde ich einzelne Themenbereiche kirchlicher Medienarbeit näher beleuchten. 


\section{Drei „Säulen” des kirchlichen Medienengagements}

Erste Säule: Engagement in Fernsehen, Hörfunk und Internet Vor dem Hintergrund einer sich im raschen Wandel befindenden Medienlandschaft beschäftigt sich die Deutsche Bischofskonferenz zurzeit auch mit der Frage, wie die katholische Kirche in einem sich fragmentierenden Fernsehmarkt künftig ihrem Kommunikationsauftrag gerecht werden kann. Dabei beobachtet sie ein verstärktes Engagement von evangelikalen Predigerkirchen, die über Satellit zunehmend im deutschsprachigen Raum zu empfangen sind und ihre Sendungen teilweise gegen traditionelle Gottesdienste programmieren. Zudem ist ein wachsender Markt von Sinnanbietern festzustellen. Weltweit reagiert die katholische Kirche auf diese Entwicklungen und wird in diesen Bestrebungen vom Päpstlichen Medienrat unterstützt. Schon heute bestehen weltweit 60 katholische Fernsehsender. Erinnert sei an den Päpstlichen Medienrat, der vor wenigen Monaten dazu aufgerufen hat, die katholischen Bemühungen in der Welt des Fernsehens mit den neuen Möglichkeiten der Digitalisierung zu bündeln.

Neben dieser Entwicklung muss mehr denn je auf die öffentlichrechtlichen Hörfunk- und Fernsehanstalten geachtet werden, die verfassungsrechtlich die Aufgabe haben, für eine umfassende Grundversorgung mit den Schwerpunkten Information, Bildung, Kultur und Unterhaltung einzutreten. Im Hörfunk haben die Kirchen mit den sogenannten Verkündigungssendungen zur Prime Time am Morgen eine besondere, rechtlich verankerte Stellung. In den Landes- und Regionalprogrammen werden kirchliche Ereignisse gerne als ästhetisch attraktives Geschehen wahrgenommen und erfreuen sich - nach Sendern und Regionen unterschiedlich - einer beachtlichen Aufmerksamkeit. Aber oft ist die "Auffindbarkeit" von Religion, Kirche und Christentum schwächer geworden. Medial verbreitete kirchliche Großereignisse wie in den vergangenen Jahren dürfen darüber nicht hinwegtäuschen. Ich denke dabei etwa an das Sterben, den Tod, das Requiem Papst Johannes Pauls II., an die Wahl des deutschen Kardinals Joseph Ratzinger zum Papst Benedikt XVI. oder an den Kölner Weltjugendtag im Jahr 2005.

Die Kirchen haben in den Programmen der öffentlich-rechtlichen Sendeanstalten eine starke Resonanz. Dabei betone ich aber: Auch bei einem möglichen eigenen kirchlichen Fernsehengagement haben die öffentlich-rechtlichen Sendeanstalten für die Kirche eine große Bedeutung. Und umgekehrt: Die öffentlich-rechtlichen Sendeanstalten haben in den Kirchen ihre stärksten Verbündeten, wenn es um die adäquate Grundversorgung, um den Kulturauftrag, um 
das Bestehen auf Seriosität und Qualität geht. Wir müssen darauf achten, dass in einer neu entstehenden digitalen religiösen TV-Szene die Deutung dessen, was Glaube, Christentum, Kirche heißt, nicht in beliebiger Weise anderen religiösen Gruppierungen überlassen wird. Es kommt darauf an, dass bei der Vielzahl von Programmen auch die katholische Kirche mit der ihr anvertrauten Botschaft auffindbar sein muss.

Entsprechende Überlegungen bedeuten also keine Abkehr von der gewachsenen Beziehung zu den öffentlich-rechtlichen Programmen. Im Gegenteil: Gerade bei einem sich zunehmend fragmentierenden Fernsehmarkt ist es notwendig, in den sogenannten Vollprogrammen der öffentlich-rechtlichen Sender weiterhin vertreten $z \mathfrak{u}$ sein. Über viele Jahre hat die katholische Kirche hierzu geeignete Strukturen aufgebaut, die auch künftig beibehalten und ausgebaut werden müssen. Ein eigenes katholisches digitales Fernsehen könnte in Verbindung mit einem weiterentwickelten Internetportal eine wichtige zusätzliche Informations- und Hinweisfunktion erfüllen. Digitales kirchliches Fernsehen kann weder in seiner inhaltlichen Gestaltung noch in seiner ästhetischen Wahrnehmbarkeit mit traditionellen Vollprogrammen öffentlich-rechtlicher Sendeanstalten vergleichbar sein.

Zu den aktuellen Entwicklungen im Medienbereich zählt auch der Aufschwung des Internets. Hier hat die katholische Kirche durch das im Frühjahr 2004 eröffnete Internetportal www.katholisch.de ein viel beachtetes Angebot entwickelt. Die stark visualisierte Seite hat den Auftrag, Wegweiser durch das katholische Deutschland zu sein, katholische Informationsangebote zu bündeln und ein zertifiziertes Suchsystem für katholische Inhalte zur Verfügung zu stellen. Es hat sich konzeptionell bewährt, die knapp 19000 redaktionell geprüften Links mit redaktionell erarbeiteten Dossiers und aktuellen Nachrichten zu ergänzen.

Durch die Anforderungen der Sinus-Milieu-Studie, durch die technischen Entwicklungen des Internets (Stichwort „Web 2.0") und durch die innerkirchlichen Ansprüche wurde ein Relaunch des Portals notwendig, der Ende 2007 abgeschlossen wurde. Zunehmend informieren sich kirchlich Distanzierte im Internet und stoßen auf www.katholisch.de. Die Redaktion reagiert täglich auf eine Vielzahl von E-Mail- und Telefonanfragen. Nach dem Relaunch ist es gelungen, noch mehr verständliche, erklärende und "niedrigschwellige“ Angebote auch für Menschen mit geringer Lesekompetenz anzubieten. Ziel dieser Kommunikation ist es, positive Erfahrungen mit der Kirche zu vermitteln. Dazu gehören auch ansprechende audiovisuelle Informationen über das katholische Leben. Es ist abzusehen, dass das Inter- 
net noch an Bedeutung gewinnen wird. Fernsehen und Internet sind in engem Zusammenhang zu sehen, weil über das Internet nicht nur ergänzende Informationen verbreitet werden können, sondern auch der individuelle Abruf von Programmen möglich wird.

\section{Zweite Säule: Gedruckte Publikationen}

Steht die Kirche bei elektronischen Medien vor der Aufgabe, neue Möglichkeiten der Kommunikation zu nutzen, geht es im herkömm. lichen Bereich gedruckter Publikationen darum, die Verbreitung religiöser bzw. kirchlich relevanter Inhalte zu verbessern. Das Internet mag zwar faszinierende Möglichkeiten bieten, aber das Jahrhunderte alte Medium Buch (einschließlich Zeitungen, Zeitschriften und anderer Druckerzeugnisse) wird weiterhin erfolgreich bestehen. Dies wird eindrucksvoll untermauert durch die Erfolge von Bestsellern wie den Harry.Potter-Bänden, aber auch von religiösen Büchern wie etwa dem "Jesus"-Buch von Papst Benedikt XVI. oder den Büchern des Benediktinerpaters Anselm Grün.

Allerdings darf das nicht darüber hinwegtäuschen, dass der konfessionelle Buchhandel zunehmend unter Druck gerät. Beeinflusst wird die Entwicklung im religiösen Buchmarkt vor allem durch das Internet. Es verändert das Einkaufsverhalten der Leserinnen und Leser und stärkt deren Individualisierung. Unverkennbar ist, dass die großen Internetbuchhändler gegenüber den Buchhändlern vor Ort an Macht und Einfluss gewinnen. Gerade kleineren und mittleren Anbietern konfessionell-religiösen Zuschnitts macht diese Entwicklung zunehmend zu schaffen. Anders als im örtlichen Buchhandel gibt es in der Verlagsszene derzeit keine dramatischen strukturellen Verwerfungen mehr, ein zunehmender Konzentrationsprozess ist aber auch hier zu festzustellen. Eine positive Sonderstellung nimmt innerhalb dieser Entwicklung der Klosterbuchhandel ein.

Die Kirche kann mit den Möglichkeiten der Digitalisierung auch die Verbreitung der gedruckten konfessionellen Publikationen fördern. Deshalb ging im Jahr 2003 mit Unterstützung der Deutschen Bischofskonferenz das Internetportal www.buchreligion.de an den Start. Zur Zeit sind dort rund 50000 religiöse Titel verzeichnet. Dieses Internetportal will helfen, neue Rezipientengruppen anzusprechen, um diese für eine christlich motivierte Literatur zu interessieren und zu gewinnen.

$\mathrm{Zu}$ den tragenden Säulen im Bereich gedruckter Publikationen gehören maßgeblich die Büchereiverbände St. Michaelsbund und Borromäusverein. Die katholischen öffentlichen Büchereien zählen zu den zentralen kulturellen Einrichtungen in Deutschland. In 22 Diözesen 
gibt es rund 3880 katholische öffentliche Büchereien, die durch die diözesanen Fachstellen und die beiden Büchereiverbände unterstützt und gefördert werden. Rund 1,4 Millionen Menschen nutzen jährlich die Einrichtungen des St. Michaelsbundes und Borromäusvereins. Einen besonderen Dienst leisten die 35000 zumeist ehrenamtlich tätigen Mitarbeiterinnen und Mitarbeiter. Sie haben im vergangenen Jahr mehr als 40000 Veranstaltungen in den katholischen öffentlichen Büchereien durchgeführt.

Die kirchliche Publizistik ist äußerst vielfältig und spricht unterschiedlichste Personengruppen an. Verweisen möchte ich exemplarisch auf die Bistumszeitungen mit einer Auflage von rund 800000 Exemplaren sowie die Ordens- und Missionspresse; ebenso auf die politische Wochenzeitung "Rheinischer Merkur/Christ und Welt", die bei Entscheidungsträgern ein hohes Renommee genießst. An eine gebildete Leserschaft, die an einer differenzierten theologischen und kirchenpolitischen Diskussion interessiert ist, wenden sich die Periodika "Herder-Korrespondenz" und "Christ in der Gegenwart". Das Spektrum theologischer Fachzeitschriften kann hier nur summarisch erwähnt werden. Nicht vergessen werden dürfen die Pfarrbriefe und die Blätter der Kirchengemeinden, Seelsorgeeinheiten und Dekanate, die mit einer Gesamtauflage von rund fünf Millionen viele Menschen erreichen. Es ist sicherlich gut investierte Mühe, auch bei diesen lokalen Publikationen eine qualitative Weiterentwicklung zu fördern.

\section{Dritte Säule: Multiplikatorenbezogene Angebote}

Multiplikatorenbezogene Angebote sind von besonderer Bedeutung. Wenn verantwortungsbewusste Journalisten in den Medien tätig sind (vielleicht sogar in verantwortlichen Positonen), die kirchlich geprägt und für religiöse Themen offen sind, dann können sie der Präsen $z$ von Glauben und Kirche in der Gesellschaft einen unschätzbaren Dienst erweisen. Sie müssen ihre Arbeit nach den Kriterien medialer Professionalität leisten sowie den internen Gegebenheiten und dem pluralen Spektrum ihrer Medieninstitutionen gerecht werden. Dennoch haben sie vielfältige Möglichkeiten der inhaltlich qualifizierten Gestaltung.

Deshalb ist eine qualifizierte und in der Fachwelt anerkannte Ausbildung des journalistischen Nachwuchses durch kirchliche Institute von höchster Bedeutung, ebenso wie die entschiedene Unterstuitzung journalistisch hochwertiger Dienstleistungen für die Medien durch kirchlich orientierte Agenturarbeit.

$\mathrm{Zu}$ den wichtigsten multiplikatorenbezogenen Angeboten gehört die Journalistenausbildung des Instituts zur Förderung publizisti- 
schen Nachwuchses e.V. (ifp). Nicht ohne Stolz können wir auf die mehr als 1000 Absolventen verweisen, die heute in zahlreichen Schlïsselpositionen im Medienbereich vertreten sind. Die Deutsche Bischofskonferenz hat sich 2005 entschieden, das ifp zukünftig in München anzusiedeln. Sie unterstützt die positive Entwicklung des Instituts nachdrücklich. Die Umbauarbeiten im ehemaligen Kapuzinerkloster St. Anton in München, das als künftiger Standort des ifp vorgesehen ist, schreiten planmäßig voran. Der Bezug des neuen Domizils ist für April dieses Jahres vorgesehen; die offizielle Einweihungsfeier wird im September stattfinden. Es ist schon jetzt spürbar, dass die Standortkonzentration neue Impulse ausgelöst hat und das ifp mit geschärftem Profil seine Erfolgsgeschichte weiter schreiben wird. Das „Medienkloster“ wird als architektonisch prägnanter Sitz des Instituts dazu beitragen, die Marke ifp weiter zu etablieren.

Auch die Katholische Nachrichtenagentur (KNA) spielt eine zentrale Rolle. Die KNA erreicht mit ihren Inhalten die Redakteure der belieferten Medien, die das Agenturangebot einerseits zur eigenen Information nutzen, vor allem aber über seine Veröffentlichung entscheiden. Die KNA steht unter einem hohen Druck: Sie muss so gut sein und als so gut eingeschätzt werden, dass ihre Angebote in die Publikationen und Sendungen Eingang finden. Sie muss eine qualifizierte und in ihrer journalistischen Kompetenz respektierte Fachagentur für Catholica sein; sie muss den Erwartungen ihrer kirchlichen Auftraggeber gerecht werden und zugleich dem Verdacht entgehen, als PR-Instrument der Kirche zu gelten und damit in der Medienlandschaft nicht ernst genommen zu werden. Die Abhängigkeit von den Entwicklungen im Medienmarkt bringt es mit sich, dass die KNA auch von den Problemen ihrer Kunden abhängig ist. Die Entwicklungen der Neuen Medien haben den Zeitungsmarkt hart getroffen. Die finanziellen Probleme der Zeitungen belasten auch die Agenturen als Zulieferer. So ist die KNA schon seit einiger Zeit dabei, sich wirtschaftlich und konzeptionell neu zu strukturieren, um den veränderten Bedingungen des Marktes gerecht zu werden. Die Deutsche Bischofskonferenz ist sich der Bedeutung der KNA bewusst. Es ist unumstritten, dass die KNA im Medienengagement der katholischen Kirche höchste Priorität genießt.

$\mathrm{Zu}$ den multiplikatorenbezogenen Dienstleistungen zählt auch die katholische Filmkritik, die in der Zeitschrift "film-dienst" ihr publizistisches Flaggschiff hat. Die Katholische Filmarbeit feierte im Oktober 2007 das 60-jährige Jubiläum ihrer renommierten Zeitschrift. Ziel dieser Filmarbeit, der "filmkulturelle" Aspekt, ist die Unterstuitzung eines qualitativ hochwertigen und vielfältigen Programms im 
filmischen Bereich (Kino, Video, Fernsehen). In medienpädagogischer Hinsicht geht es um einen originären Beitrag zur Ausbildung von Medienkompetenz.

Eine mit der Filmkritik eng verbundene Aktivität ist die Präsenz von ökumenischen Jurys auf internationalen Filmfestivals. Auch hier geht es darum, Filme zu beurteilen und solche Werke durch Preise zu unterstuitzen, die aus christlicher Sicht relevante Themen in eine überzeugende künstlerische Form zu bringen vermögen. In Deutschland steht die Berlinale als wichtigstes Festival im Mittelpunkt. Dort ist seit gut fünf Jahrzehnten eine kirchliche Jury vertreten.

Seit einigen Jahren konnten der Katholische Kinder- und Jugendbuchpreis sowie die Neugestaltung des Katholischen Medienpreises erfolgreich etabliert werden. Eine gezielte Kontaktpflege zu den Verlagen hat bewirkt, dass die Beteiligung beim Katholischen Kinder- und Jugendbuchpreis auf ein hohes quantitatives und qualitatives Niveau geführt werden konnte. Nach Einschätzung von Buchexperten genießen der Katholische Kinder- und Jugendbuchpreis und der Deutsche Jugendliteraturpreis inzwischen ein vergleichbares Renommee.

Ein ähnlich positives Fazit lässt sich mit Blick auf die Neugestaltung des Katholischen Medienpreises ziehen. Er hat ein eigenständiges Profil erhalten. Die Wahrnehmung dieses Preises sowohl in der konfessionellen als auch in der säkularen Medienszene ist dadurch deutlich gestiegen.

Einen weiteren Baustein von Diensten in der Multiplikatorenarbeit stellt der medien- und kommunikationspädagogische Zertifikatskurs "Medientutor/Medientutorin" dar. Hierbei handelt es sich, in Kooperation mit namhaften Veranstaltern, um eine jährliche Kursreihe zur Ausbildung von katholischen Medienpädagogen. Durch eine ausgewogene Mischung von Theorie und Praxis erweitern die Teilnehmer im Bezug auf Medien ihre philosophische, psychologische und theologische Reflexionsfähigkeit und werden sensibel für die besondere kirchliche Medienverantwortung.

\section{Fazit}

Die Bandbreite kirchlicher Medienarbeit ist sehr vielfältig. Alles hat hier seine spezifische Geschichte und Berechtigung. Allerdings muss es auch um Prioritätensetzungen und -entscheidungen gehen. Diese bedürfen wegen der sachlich bedingten Schwierigkeiten und der jeweils bestehenden Interessen einer sorgfältigen Abwägung und eines sensiblen Diskussionsprozesses. Derzeit kann man Folgendes festhalten: Der Journalistenausbildung und der Stärkung der Katholischen 
Nachrichtenagentur wird eine ïbergeordnete Bedeutung beigemessen. Diese Engagements sind eine unabdingbare Voraussetzung für ein langfristig angemessenes Wirken der katholischen Kirche in den Medien. Fernsehen, Hörfunk und Internet unterliegen einem dynamischen Wandlungsprozess. Um anschlussfähig zu bleiben, müssen hier neue Schwerpunktsetzungen nach sorgfältiger Prüfung umgesetzt werden. Der Bereich gedruckter kirchlicher Publikationen bleibt eine klassische Säule kirchlicher Medienarbeit.

Als Fazit möchte ich drei Punkte festhalten:

Erstens: Die Kirche muss sowohl die kirchennahen als auch kirchenferne Personengruppen erreichen. Auch wenn sie nicht alle Bevölkerungsgruppen ansprechen kann, muss sie doch bemüht sein, sich nicht nur an die ohnehin schon an die Kirche Gebundenen zu wenden, sondern auch an solche Gruppen, die der Kirche distanziert gegenüberstehen. Es ist von entscheidender Bedeutung, die Offenheit und das Interesse von Personen zu finden, die maßgeblich das gesellschaftliche Engagement und die öffentliche Meinung prägen. Wïrde die Kirche mit ihren medialen Angeboten nur noch kirchlich identifizierte Menschen erreichen, würde sie ihrem Auftrag nicht gerecht.

Zweitens: Die Kirche muss für die verschiedenen Möglichkeiten der medialen Ansprache offen sein. Sie muss die heute gegebenen Möglichkeiten der Medien nutzen. Das bedingt, dass die Kirche in unterschiedlichen Programmformaten unterschiedlich auftreten muss. Information verlangt andere Herangehensweisen als Glaubensvermittlung oder Unterhaltung.

Drittens: Die Kirche muss im Hinblick auf die Kommunikationskanäle eine diversifizierte Strategie verfolgen. Als eine traditionell stark der Schriftkultur verbundene Institution hat sie eine starke Bindung an gedruckte Publikationen. Sie muss aber auch die neuen Technologien nutzen, um den Interessen und Kommunikationsgewohnheiten in einer modernen Gesellschaft gerecht $\mathrm{zu}$ werden. So muss es Ziel der kirchlichen Medienstrategie sein, nicht nur einseitig auf eine Form medialer Kommunikation zu setzen, sondern die erforderliche Bandbreite an Möglichkeiten kirchlicher Kommunikationsarbeit im Blick zu behalten. Das bedeutet, Bewährtes zu unterstützen und zu optimieren, aber gleichzeitig die neuen Möglichkeiten zu nutzen. 